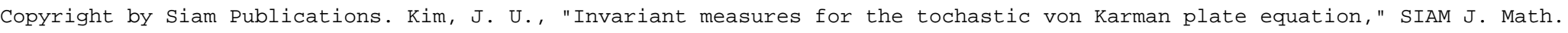
Anal., 36(5), 1689-1703, (2005). DOI: 10.1137/s0036141003438854

SIAM J. MATH ANAL.

Vol. 36 , No. 5 , pp. $1689-1703$

(c) 2005 Society for Industrial and Applied Mathematics

\title{
INVARIANT MEASURES FOR THE STOCHASTIC VON KARMAN PLATE EQUATION*
}

\author{
JONG UHN KIM ${ }^{\dagger}$
}

\begin{abstract}
We prove the existence of an invariant measure for the von Karman plate equation with random noise. The nonlinear term which symbolizes the von Karman equation inhibits the standard procedure for the existence of an invariant measure. We propose a technically different approach to handle such intricate nonlinear equations.
\end{abstract}

Key words. von Karman plate equation, Brownian motion, stopping time, existence of a solution, invariant measure, probability distribution, tightness

AMS subject classifications. 35L65, 35R60, 60H15

DOI. $10.1137 / \mathrm{S} 0036141003438854$

1. Introduction. In this paper, we will establish the existence of an invariant measure for a certain class of stochastic evolution equations with application to the stochastic von Karman plate equation. An invariant measure is an important object in stochastic dynamics. If the initial condition has the probability distribution equal to an invariant measure, then the probability distribution of the evolving solution is invariant in time. Some general results on the existence of invariant measures for stochastic evolution equations are presented in [6] and [7]. The basic method for the existence of invariant measures is due to Krylov and Bogolyubov [12]. However, there are some important equations which are not covered by the known theorems. Here we still follow the Krylov-Bogolyubov method, but with technically different adaptation, which has been motivated by the von Karman equation. For our method, we assume that the stochastic process associated with solutions has the Markov property with mean energy bounded uniformly in time, and that the probability distribution of the process is locally continuous with respect to a weaker norm. Typically, the first assumption is satisfied by a wide class of stochastic evolution equations with suitable dissipation. However, we need an additional condition for tightness of a family of probability measures which will yield an invariant measure. For parabolic equations, the regularizing property is crucially used to obtain tightness of a sequence of probability measures whose weak limit is an invariant measure; see [2]. Hyperbolic equations do not possess the regularizing property. But if the noise term has additional regularity in space variables and if more regular initial data can generate more regular solutions with a higher-order norm bounded uniformly in time, tightness of probability measures can be obtained in the same manner. There are equations which belong to neither case. The von Karman plate equation is a typical example. The advantage of this proposed approach lies in the second assumption, which is fairly mild and can be satisfied by equations such as the von Karman equation. We will highlight the utility of this procedure through the specific example of the von Karman equation.

The initial-boundary value problem for the von Karman plate is formulated as

* Received by the editors December 22, 2003; accepted for publication (in revised form) August 24, 2004; published electronically May 13, 2005.

http://www.siam.org/journals/sima/36-5/43885.html

${ }^{\dagger}$ Department of Mathematics, Virginia Tech, Blacksburg, VA 24061-0123 (kim@math.vt.edu). 
follows:

$$
\begin{gathered}
u_{t t}+\alpha u_{t}+\Delta^{2} u-[u, v]=\sum_{j=1}^{\infty} g_{j} \frac{d B_{j}}{d t} \quad \text { in }(0, T) \times G, \\
\Delta^{2} v+[u, u]=0 \quad \text { in }(0, T) \times G, \\
u=\frac{\partial u}{\partial \nu}=0, \quad v=\frac{\partial v}{\partial \nu}=0 \quad \text { on }[0, T] \times \partial G, \\
u=u_{0}(x), \quad u_{t}=u_{1}(x) \quad \text { at } t=0 .
\end{gathered}
$$

Here $G$ is a bounded domain in $R^{2}$ with smooth boundary $\partial G, \Delta$ is the Laplacian in $R^{2}, \frac{\partial}{\partial \nu}$ is the normal derivative on $\partial G$, and the bracket $[\cdot, \cdot]$ is defined by

$$
[u, v]=\frac{\partial^{2} u}{\partial x^{2}} \frac{\partial^{2} v}{\partial y^{2}}+\frac{\partial^{2} v}{\partial x^{2}} \frac{\partial^{2} u}{\partial y^{2}}-2 \frac{\partial^{2} u}{\partial x \partial y} \frac{\partial^{2} v}{\partial x \partial y} .
$$

Viscous damping is represented by a positive constant $\alpha$, and $B_{j}$ 's are mutually independent standard Brownian motions over a given stochastic basis. When the righthand side of (1.1) is replaced by a deterministic term, the existence of a weak solution to (1.1)-(1.4) was proved in [15], and more regular solutions were obtained in [4] and [8]. In fact, the weak solution belongs to the natural function class. Nevertheless, the uniqueness of the weak solution had been an open problem until the work of [1] and [8]. The existence and uniqueness of a solution to the stochastic problem (1.1)-(1.4) can be proved through a standard procedure based upon the known results from the deterministic case. The existence of statistical solutions was established in [3] and [10]. At present, the significant issue is the existence of an invariant measure.

Plate equations are neither hyperbolic nor parabolic while there is no regularizing property. In [4], it was shown that for large $\alpha>0$ depending on the magnitudes of the given data, the bound of the global solution in a stronger norm is uniform in time. However, for small $\alpha>0$, it is not known whether such an estimate is valid. Probably, it may not be true. This feature puts the above problem in a new category, which necessitates a technically different approach. Here we proceed in the opposite direction. Instead of trying to find uniform estimates in a stronger norm, we imbed the natural energy space into a larger function class, and obtain a probability measure on this larger space as a limit of a tight family of probability measures. We then prove that this is in fact an invariant measure on the original smaller space. For this, we need to show that the probability distribution of the solution depends continuously on initial data in a weaker norm for fixed time on each closed ball in the natural energy space. The main advantage of this procedure is that we do not need any additional estimates uniform in time other than uniform estimates in the natural energy space. Hence, we do not need either the assumption that $\alpha>0$ is large or additional regularity of the noise term. We expect this procedure to be applied to other equations which behave like (1.1). Finally, the anonymous referee has informed the author that the idea of using a weaker topology was already used for interacting diffusions in [14] and for stochastic parabolic equations in [16] and [17].

2. Existence of invariant measures. Let $\left\{\Omega, \mathcal{F}_{t}, \mathcal{F}, P\right\}$ be a given stochastic basis and let $E(\cdot)$ denote the expectation with respect to $P$. Suppose that $X(t, s ; z), 0 \leq$ $s \leq t<\infty$ is a pathwise unique solution of a certain stochastic evolution equation such that $X(s, s ; z)=z$. We assume

(I) $X(\cdot, s ; z)$ is a $\Xi$-valued continuous process adapted to $\left\{\mathcal{F}_{t}\right\}_{t \geq s}$ for each $z \in \Xi$ and $s \geq 0$, where $\Xi$ is a separable Banach space. 
We define a function

$$
\mathcal{P}(s, z ; t, \Gamma)=P(X(t, s ; z) \in \Gamma) \quad \text { for each } \Gamma \in \mathcal{B}(\Xi), 0 \leq s \leq t<\infty, z \in \Xi,
$$

where $\mathcal{B}(\Xi)$ is the Borel $\sigma$-algebra of $\Xi$. We assume

(II) $\mathcal{P}(\cdot, \cdot ; \cdot, \cdot)$ is a time-homogeneous transition probability function. In other words, it satisfies the following conditions:

(i) $\mathcal{P}(s, z ; t, \cdot)$ is a probability measure over $\{\Xi, \mathcal{B}(\Xi)\}$ for all $z \in \Xi$ and $0 \leq$ $s<t<\infty$

(ii) $\mathcal{P}(s, \cdot ; t, \Gamma)$ is $\mathcal{B}(\Xi)$-measurable for all $0 \leq s<t<\infty$ and $\Gamma \in \mathcal{B}(\Xi)$;

(iii) for all $0 \leq s<t<\xi<\infty$ and $\Gamma \in \mathcal{B}(\Xi)$,

$$
\mathcal{P}(s, z ; \xi, \Gamma)=\int_{\Xi} \mathcal{P}(s, z ; t, d y) \mathcal{P}(t, y ; \xi, \Gamma) ;
$$

(iv) $\mathcal{P}(s, \cdot ; t, \cdot)=\mathcal{P}(s+h, \cdot ; t+h, \cdot)$ for all $0 \leq s<t<\infty$ and $h>0$.

(III) There is some $z \in \Xi$ such that

$$
E\left(\|X(t, 0 ; z)\|_{\Xi}\right) \leq M \text { for all } t \geq 0
$$

for some positive constant $M$.

(IV) There is a Banach space $\Upsilon$ such that $\Xi \subset \Upsilon$, the imbedding $\Xi \rightarrow \Upsilon$ is continuous, and each closed ball of finite radius in $\Xi$ is a compact subset of $\Upsilon$. Furthermore, for each bounded continuous function $\psi$ on $\Xi$, there is a sequence of continuous functions $\left\{\psi_{k}\right\}_{k=1}^{\infty}$ on $\Upsilon$ such that $\psi_{k}$ is bounded uniformly in $k$ and

$$
\lim _{k \rightarrow \infty} \psi_{k}(y)=\psi(y) \quad \text { for each } y \in \Xi .
$$

(V) For each fixed $0 \leq t<\infty$, and each fixed closed ball $S$ of finite radius in $\Xi$, if $\left\{z_{n}\right\}_{n=1}^{\infty}$ is a sequence in $S$ such that

$$
z_{n} \rightarrow z \quad \text { in } \Upsilon
$$

then

$$
E\left(\phi\left(X\left(t, 0 ; z_{n}\right)\right)\right) \rightarrow E(\phi(X(t, 0 ; z)))
$$

for every bounded continuous function $\phi$ on $\Upsilon$.

Remark. If $\Xi$ has a Schauder basis, the second part of assumption (IV) is automatically satisfied by using the continuous projection onto finite-dimensional subspaces. In fact, this is the case when we consider application to the von Karman plate equation.

THEOREM 2.1. Under the assumptions $(\mathrm{I})-(\mathrm{V})$, there is an invariant measure for the above process $X(\cdot)$. In other words, there is a probability measure $\mu$ on $\Xi$ such that

$$
\int_{\Xi} E(\psi(X(t, 0 ; y))) \mu(d y)=\int_{\Xi} \psi(y) \mu(d y)
$$

for all $t \geq 0$ and every bounded continuous function $\psi$ on $\Xi$.

Proof. Choose $z \in \Xi$ in the above assumption (III), and define a probability measure $\mu_{T}$ for each $T>0$ by

$$
\mu_{T}(\Gamma)=\frac{1}{T} \int_{0}^{T} P(X(t, 0 ; z) \in \Gamma) d t
$$


for each $\Gamma \in \mathcal{B}(\Xi)$. This is well defined because $P(X(\cdot, 0 ; z) \in \Gamma)$ is $\mathcal{B}([0, \infty))$ measurable. For this measurability, we argue as follows. For each bounded continuous function $\phi$ on $\Xi, E(\phi(X(t, 0 ; z)))$ is continuous in $t$ by assumption (I). Let $\Gamma$ be a closed subset of $\Xi$ and $\chi_{\Gamma}(\cdot)$ be the characteristic function of $\Gamma$. Then, there is a sequence of nonnegative bounded continuous functions $\left\{\phi_{k}\right\}_{k=1}^{\infty}$ on $\Xi$ such that $\phi_{k}(y) \downarrow \chi_{\Gamma}(y)$ as $k \rightarrow \infty$ for each $y \in \Xi$. Hence, $E\left(\phi_{k}(X(t, 0 ; z))\right)$ converges to $E\left(\chi_{\Gamma}(X(t, 0 ; z))\right)$ as $k \rightarrow \infty$ for each $t$. Hence, $P(X(\cdot, 0 ; z) \in \Gamma)$ is $\mathcal{B}([0, \infty))$-measurable. Let $\mathcal{S}$ be the collection of all subsets $\Gamma$ such that $P(X(\cdot, 0 ; z) \in \Gamma)$ is $\mathcal{B}([0, \infty))$-measurable. Then, $\mathcal{S}$ is a Dynkin system which includes all closed subsets of $\Xi$. Thus, $\mathcal{S}$ contains $\mathcal{B}(\Xi)$.

We now proceed to define

$$
\tilde{\mu}_{T}(\Gamma)=\mu_{T}(\Gamma \cap \Xi)
$$

for each $\Gamma \in \mathcal{B}(\Upsilon)$. Since the imbedding $\Xi \rightarrow \Upsilon$ is continuous, $\Gamma \cap \Xi$ is a Borel subset of $\Xi$ for each $\Gamma \in \mathcal{B}(\Upsilon)$. Hence, $\tilde{\mu}_{T}$ is well defined and is a probability measure over $\{\Upsilon, \mathcal{B}(\Upsilon)\}$. For any $\epsilon>0$, there is a positive number $r_{\epsilon}$ such that

$$
P\left(\|X(t, 0 ; z)\|_{\Xi} \leq r_{\epsilon}\right)>1-\epsilon \text { for all } t \geq 0
$$

which follows from assumption (III). Since the ball

$$
S_{r_{\epsilon}}=\left\{y \in \Xi \mid\|y\|_{\Xi} \leq r_{\epsilon}\right\}
$$

is a compact subset of $\Upsilon$ by assumption (IV), the family of probability measures $\left\{\tilde{\mu}_{T}\right\}_{T>0}$ is tight. Hence, there is a sequence $\left\{\tilde{\mu}_{T_{k}}\right\}_{k=1}^{\infty}$ and a probability measure $\tilde{\mu}$ over $\{\Upsilon, \mathcal{B}(\Upsilon)\}$ such that $T_{k} \uparrow \infty$ as $k \rightarrow \infty$, and

$$
\int_{\Upsilon} \phi(y) \tilde{\mu}_{T_{k}}(d y) \rightarrow \int_{\Upsilon} \phi(y) \tilde{\mu}(d y) \quad \text { as } k \rightarrow \infty
$$

for every bounded continuous function $\phi$ on $\Upsilon$. Since $S_{r_{\epsilon}}$ is a closed subset of $\Upsilon$, it follows from (2.9) that

$$
1-\epsilon \leq \limsup _{k \rightarrow \infty} \tilde{\mu}_{T_{k}}\left(S_{r_{\epsilon}}\right) \leq \tilde{\mu}\left(S_{r_{\epsilon}}\right) .
$$

Since $\epsilon>0$ is arbitrary and each Borel subset of $\Xi$ is also a Borel subset of $\Upsilon, \tilde{\mu}(\Xi)=1$ and the restriction of $\tilde{\mu}$ to $\mathcal{B}(\Xi)$, written as $\mu$, is a probability measure over $\{\Xi, \mathcal{B}(\Xi)\}$. Choose any bounded continuous function $\phi$ on $\Upsilon$, and fix any $\epsilon>0$. Then, there is $r>0$ such that

$$
\tilde{\mu}_{T_{k}}\left(S_{r}\right)=\mu_{T_{k}}\left(S_{r}\right)>1-\epsilon \text { for all } k \geq 1 .
$$

Fix $t>0$, and let

$$
f(y)=E(\phi(X(t, 0 ; y)))=\int_{\Xi} \mathcal{P}(0, y ; t, d w) \phi(w) .
$$

Then, by assumption $(\mathrm{V}), f(y)$ is continuous on $S_{r}$ with respect to the norm of $\Upsilon$. Since $S_{r}$ is a closed subset of $\Upsilon$, we can extend $f$ to $\tilde{f}$ on $\Upsilon$ with the same bound such that $f(y)=\tilde{f}(y)$ for every $y \in S_{r}$. This follows from the Tietze extension theorem.

It is easy to see that

$$
\begin{aligned}
\int_{\Upsilon} \tilde{f}(y) \tilde{\mu}_{T_{k}}(d y)= & \int_{\Upsilon \backslash S_{r}} \tilde{f}(y) \tilde{\mu}_{T_{k}}(d y) \\
& +\int_{S_{r}} \tilde{\mu}_{T_{k}}(d y) \int_{\Xi} \mathcal{P}(0, y ; t, d w) \phi(w)
\end{aligned}
$$


and, by (2.13),

$$
\left|\int_{S_{r}} \tilde{\mu}_{T_{k}}(d y) \int_{\Xi} \mathcal{P}(0, y ; t, d w) \phi(w)-\int_{\Xi} \tilde{\mu}_{T_{k}}(d y) \int_{\Xi} \mathcal{P}(0, y ; t, d w) \phi(w)\right|<M \epsilon,
$$

where $M$ is a positive constant such that $|\phi(y)| \leq M$, for all $y \in \Upsilon$. Here we note that $\phi$ is also a continuous function on $\Xi$ with respect to the norm of $\Xi$. It follows from assumption (II) that

$$
\begin{aligned}
\int_{\Xi} & \mu_{T_{k}}(d y) \int_{\Xi} \mathcal{P}(0, y ; t, d w) \phi(w) \\
& =\frac{1}{T_{k}} \int_{0}^{T_{k}}\left(\int_{\Xi} \mathcal{P}(0, z ; s, d y) \int_{\Xi} \mathcal{P}(0, y ; t, d w) \phi(w)\right) d s \\
& =\frac{1}{T_{k}} \int_{0}^{T_{k}}\left(\int_{\Xi} \mathcal{P}(0, z ; s+t, d y) \phi(y)\right) d s \\
& =\frac{1}{T_{k}} \int_{t}^{T_{k}+t}\left(\int_{\Xi} \mathcal{P}(0, z ; \eta, d y) \phi(y)\right) d \eta .
\end{aligned}
$$

But we have

$$
\begin{gathered}
\lim _{k \rightarrow \infty}\left|\frac{1}{T_{k}} \int_{t}^{T_{k}+t}\left(\int_{\Xi} \mathcal{P}(0, z ; \eta, d y) \phi(y)\right) d \eta-\int_{\Xi} \mu_{T_{k}}(d y) \phi(y)\right|=0, \\
\int_{\Xi} \mu_{T_{k}}(d y) \phi(y)=\int_{\Upsilon} \tilde{\mu}_{T_{k}}(d y) \phi(y),
\end{gathered}
$$

and

$$
\lim _{k \rightarrow \infty} \int_{\Upsilon} \tilde{\mu}_{T_{k}}(d y) \phi(y)=\int_{\Upsilon} \tilde{\mu}(d y) \phi(y)=\int_{\Xi} \mu(d y) \phi(y) .
$$

In the meantime, it holds that

$$
\begin{gathered}
\lim _{k \rightarrow \infty} \int_{\Upsilon} \tilde{f}(y) \tilde{\mu}_{T_{k}}(d y)=\int_{\Upsilon} \tilde{f}(y) \tilde{\mu}(d y), \\
\left|\int_{\Upsilon} \tilde{f}(y) \tilde{\mu}(d y)-\int_{S_{r}} f(y) \mu(d y)\right|<M \epsilon, \\
\int_{S_{r}} f(y) \mu(d y)=\int_{S_{r}} \mu(d y) E(\phi(X(t, 0 ; y))),
\end{gathered}
$$

and

$$
\left|\int_{S_{r}} \mu(d y) E(\phi(X(t, 0 ; y)))-\int_{\Xi} \mu(d y) E(\phi(X(t, 0 ; y)))\right|<M \epsilon .
$$

Thus, it follows from (2.15)-(2.24) that

$$
\left|\int_{\Xi} \mu(d y) E(\phi(X(t, 0 ; y)))-\int_{\Xi} \mu(d y) \phi(y)\right|<4 M \epsilon .
$$

Since $\epsilon>0$ is arbitrary, we have

$$
\int_{\Xi} \mu(d y) E(\phi(X(t, 0 ; y)))=\int_{\Xi} \mu(d y) \phi(y)
$$


for all bounded continuous function $\phi$ on $\Upsilon$ for each $t>0$. Next choose any bounded continuous function $\psi$ on $\Xi$, and let $\left\{\psi_{k}\right\}_{k=1}^{\infty}$ be the sequence in assumption (IV). Then, for each $k \geq 1$, we have

$$
\int_{\Xi} \mu(d y) E\left(\psi_{k}(X(t, 0 ; y))\right)=\int_{\Xi} \mu(d y) \psi_{k}(y) .
$$

By passing $k \rightarrow \infty$, we have

$$
\int_{\Xi} \mu(d y) E(\psi(X(t, 0 ; y)))=\int_{\Xi} \mu(d y) \psi(y)
$$

This completes the proof.

3. Application to the stochastic von Karman equation. In this section, we present technical preliminaries to apply Theorem 2.1 to (1.1)-(1.4) and formulate the results.

Let $\left\{\phi_{k}\right\}_{k=1}^{\infty}$ be a complete orthonormal basis for $L^{2}(G)$ where each $\phi_{k}$ is an eigenfunction of

$$
\begin{cases}\Delta^{2} \phi_{k}=\lambda_{k} \phi_{k} & \text { in } G, \\ \phi_{k}=\frac{\partial \phi_{k}}{\partial \nu}=0 & \text { on } \partial G .\end{cases}
$$

Throughout this paper, $\langle\cdot, \cdot\rangle$ stands for the inner product of $L^{2}(G)$. It is easy to see that

$$
\left\langle\Delta^{2} \phi_{j}, \phi_{k}\right\rangle=\left\langle\Delta \phi_{j}, \Delta \phi_{k}\right\rangle=\lambda_{j} \delta_{j k} \quad \text { for all } j, k \geq 1 .
$$

$W^{m, p}(G), H^{m}(G)$, and $H_{0}^{m}(G)$ denote the usual Sobolev spaces. Some of them can be characterized in terms of $\left\{\phi_{k}\right\}_{k=1}^{\infty}$ :

$$
\begin{aligned}
H_{0}^{2}(G) \cap H^{4}(G) & =\left\{f=\left.\sum_{k=1}^{\infty} a_{k} \phi_{k}\left|\sum_{k=1}^{\infty} \lambda_{k}^{2}\right| a_{k}\right|^{2}<\infty\right\} \\
H_{0}^{s}(G) & =\left\{f=\left.\sum_{k=1}^{\infty} a_{k} \phi_{k}\left|\sum_{k=1}^{\infty} \lambda_{k}^{s / 2}\right| a_{k}\right|^{2}<\infty\right\}, 0 \leq s \leq 2, s \neq \frac{1}{2}, \frac{3}{2} \\
H^{-s}(G) & =\left\{f=\left.\sum_{k=1}^{\infty} a_{k} \phi_{k}\left|\sum_{k=1}^{\infty} \frac{1}{\lambda_{k}^{s / 2}}\right| a_{k}\right|^{2}<\infty\right\}, 0 \leq s \leq 2, s \neq \frac{1}{2}, \frac{3}{2}
\end{aligned}
$$

We define the operator $\mathcal{G}$ on $H^{-2}(G)$ by

$$
\mathcal{G} h=\sum_{k=1}^{\infty} \frac{1}{\lambda_{k}} a_{k} \phi_{k}
$$

for $h=\sum_{k=1}^{\infty} a_{k} \phi_{k} \in H^{-2}(G)$. Obviously, $\mathcal{G}$ is the inverse of $\Delta^{2}$ with the clamped boundary conditions. It is easy to see that for all $f, g \in L^{2}(G)$,

$$
|\langle f, \mathcal{G} g\rangle| \leq\|f\|_{H^{-2}(G)}\|g\|_{H^{-2}(G)}
$$

and

$$
\langle f, \mathcal{G} f\rangle=\|f\|_{H^{-2}(G)}^{2} .
$$


The following estimate was proved in [5] and [9]:

$$
\|\mathcal{G}[f, g]\|_{W^{2, \infty}(G)} \leq C\|f\|_{H^{2}(G)}\|g\|_{H^{2}(G)}
$$

for all $f, g \in H^{2}(G)$.

Throughout this paper, $\left\{B_{j}\right\}_{j=1}^{\infty}$ is a sequence of mutually independent standard Brownian motions over the stochastic basis $\left\{\Omega, \mathcal{F}, \mathcal{F}_{t}, P\right\}$, where $P$ is a probability measure over the $\sigma$-algebra $\mathcal{F},\left\{\mathcal{F}_{t}\right\}$ is a right-continuous filtration over $\mathcal{F}$, and $\mathcal{F}_{0}$ contains all $P$-negligible sets. $E(\cdot)$ denotes the expectation with respect to $P$. When $\mathcal{X}$ is a Banach space, $\mathcal{B}(\mathcal{X})$ denotes the set of all Borel subsets of $\mathcal{X}$. An $\mathcal{X}$-valued function $h$ is said to be $\mathcal{F}$-measurable if $h^{-1}(\mathcal{O}) \in \mathcal{F}$ for all $\mathcal{O} \in \mathcal{B}(\mathcal{X})$. This coincides with strong measurability for Bochner integrals when the range of $h$ is separable. For $1 \leq p<\infty, L^{p}(\Omega ; \mathcal{X})$ denotes the set of all functions $h$ which are $\mathcal{X}$-valued and strongly measurable with respect to $\mathcal{F}$ such that

$$
\int_{\Omega}\|h\|_{\mathcal{X}}^{p} d P<\infty
$$

For general information on stochastic processes, see [11].

We assume the following condition on the noise term in (1.1). Each $g_{j}$ depends only on the space variables, and

$$
\sum_{j=1}^{\infty}\left\|g_{j}\right\|_{L^{2}(G)}^{2}<\infty .
$$

Under this assumption, we have the following existence result.

Theorem 3.1. For each $T>0$ and $\left(u_{0}, u_{1}\right) \in H_{0}^{2}(G) \times L^{2}(G)$, there is a unique solution $u$ of (1.1)-(1.4) such that $\left(u, u_{t}\right)$ is adapted to $\left\{\mathcal{F}_{t}\right\}$, and

$$
\left(u, u_{t}\right) \in L^{2}\left(\Omega ; C\left([0, T] ; H_{0}^{2}(G) \times L^{2}(G)\right)\right) .
$$

Here $u$ satisfies (1.1) in the following sense. For almost all $\omega \in \Omega$, it holds that

$$
\begin{aligned}
& \left\langle u_{t}\left(t_{2}\right), \psi\right\rangle-\left\langle u_{t}\left(t_{1}\right), \psi\right\rangle+\int_{t_{1}}^{t_{2}}\langle\Delta u, \Delta \psi\rangle d t \\
& \quad+\alpha \int_{t_{1}}^{t_{2}}\left\langle u_{t}, \psi\right\rangle d t+\int_{t_{1}}^{t_{2}}\langle[u, \mathcal{G}[u, u]], \psi\rangle d t=\sum_{j=1}^{\infty} \int_{t_{1}}^{t_{2}}\left\langle g_{j}, \psi\right\rangle d B_{j}
\end{aligned}
$$

for all $\psi \in H_{0}^{2}(G)$ and all $0 \leq t_{1}<t_{2} \leq T$.

TheOREM 3.2. There is an invariant measure on $H_{0}^{2}(G) \times L^{2}(G)$ for (1.1)-(1.4).

4. Proof of Theorems 3.1 and 3.2. Let us define $\chi_{N} \in C_{0}^{\infty}(R)$ for each $N \geq 1$ by

$$
\chi_{N}(y)= \begin{cases}1 & \text { for }|y| \leq 2 N \\ 0 & \text { for }|y| \geq 3 N\end{cases}
$$

Then, it follows from (3.9) that

$$
\begin{aligned}
& \left\|\chi_{N}\left(\|u\|_{H^{2}(G)}\right)[u, \mathcal{G}[u, u]]-\chi_{N}\left(\|w\|_{H^{2}(G)}\right)[w, \mathcal{G}[w, w]]\right\|_{L^{2}(G)} \\
& \quad \leq C_{N}\|u-w\|_{H^{2}(G)}
\end{aligned}
$$


for all $u, w \in H^{2}(G)$ and for some positive constant $C_{N}$. We now fix $N \geq 1$ and consider the modified problem

$$
\begin{gathered}
u_{t t}+\alpha u_{t}+\Delta^{2} u+\chi_{N}\left(\|u\|_{H_{0}^{2}(G)}\right)[u, \mathcal{G}[u, u]]=\sum_{j=1}^{\infty} g_{j} \frac{d B_{j}}{d t} \quad \text { in }(0, T) \times G \\
u=\frac{\partial u}{\partial \nu}=0 \quad \text { on }[0, T] \times \partial G \\
u=u_{0}(x), \quad u_{t}=u_{1}(x) \quad \text { at } t=0 .
\end{gathered}
$$

By the general existence theorem in [6], for each $T>0$ and $\left(u_{0}, u_{1}\right) \in H_{0}^{2}(G) \times L^{2}(G)$, there is a pathwise unique solution $u$ of $(4.3)-(4.5)$ such that $\left(u, u_{t}\right)$ is adapted to $\left\{\mathcal{F}_{t}\right\}$, and

$$
\left(u, u_{t}\right) \in L^{2}\left(\Omega ; C\left([0, T] ; H_{0}^{2}(G) \times L^{2}(G)\right)\right) .
$$

This is still true when $\left(u_{0}, u_{1}\right)$ is $\mathcal{F}_{0}$-measurable and $\left(u_{0}, u_{1}\right) \in L^{2}\left(\Omega ; H_{0}^{2}(G) \times L^{2}(G)\right)$, which follows from Kotelenez [13].

We introduce the projection $P_{m}$ of $L^{2}(G)$ onto the subspace that is spanned by $\left\{\phi_{1}, \ldots, \phi_{m}\right\}$, and set

$$
u_{m}=P_{m} u \text {. }
$$

By taking the nonlinear term as a given function, we use the argument in [6, pp. 121123] to obtain the following representation formula. For almost all $\omega \in \Omega$,

$$
\begin{aligned}
& \left\langle u_{t}\left(t_{2}\right), \psi\right\rangle-\left\langle u_{t}\left(t_{1}\right), \psi\right\rangle+\int_{t_{1}}^{t_{2}}\langle\Delta u, \Delta \psi\rangle d t+\alpha \int_{t_{1}}^{t_{2}}\left\langle u_{t}, \psi\right\rangle d t \\
& \quad+\int_{t_{1}}^{t_{2}}\left\langle\chi_{N}\left(\|u\|_{H_{0}^{2}(G)}\right)[u, \mathcal{G}[u, u]], \psi\right\rangle d t=\sum_{j=1}^{\infty} \int_{t_{1}}^{t_{2}}\left\langle g_{j}, \psi\right\rangle d B_{j}
\end{aligned}
$$

for all $\psi \in H_{0}^{2}(G)$ and all $0 \leq t_{1}<t_{2} \leq T$. Thus, it follows that

$$
\begin{aligned}
d\left(u_{m t}\right)= & \left(-\Delta^{2} u_{m}-\alpha u_{m t}-\chi_{N}\left(\|u\|_{H_{0}^{2}(G)}\right) P_{m}[u, \mathcal{G}[u, u]]\right) d t \\
& +\sum_{j=1}^{\infty} P_{m} g_{j} d B_{j} \quad \text { for each } m \geq 1 .
\end{aligned}
$$

By Ito's rule, we have, for all $0 \leq t_{1}<t_{2} \leq T$ and $m \geq 1$,

$$
\begin{aligned}
&\left\|u_{m t}\left(t_{2}\right)\right\|_{L^{2}(G)}^{2}+\left\|\Delta u_{m}\left(t_{2}\right)\right\|_{L^{2}(G)}^{2} \\
&=\left\|u_{m t}\left(t_{1}\right)\right\|_{L^{2}(G)}^{2}+\left\|\Delta u_{m}\left(t_{1}\right)\right\|_{L^{2}(G)}^{2}-2 \alpha \int_{t_{1}}^{t_{2}}\left\|u_{m t}\right\|_{L^{2}(G)}^{2} d t \\
&-2 \int_{t_{1}}^{t_{2}}\left\langle\chi_{N}\left(\|u\|_{H_{0}^{2}(G)}\right) P_{m}[u, \mathcal{G}[u, u]], u_{m t}\right\rangle d t \\
&+2 \sum_{j=1}^{\infty} \int_{t_{1}}^{t_{2}}\left\langle P_{m} g_{j}, u_{m t}\right\rangle d B_{j}+\sum_{j=1}^{\infty} \int_{t_{1}}^{t_{2}}\left\|P_{m} g_{j}\right\|_{L^{2}(G)}^{2} d t .
\end{aligned}
$$

It follows from (3.9) that

$$
\begin{aligned}
&\left\|\chi_{N}\left(\|u\|_{H_{0}^{2}(G)}\right) P_{m}[u, \mathcal{G}[u, u]]-\chi_{N}\left(\|u\|_{H_{0}^{2}(G)}\right)\left[u_{m}, \mathcal{G}\left[u_{m}, u_{m}\right]\right]\right\|_{L^{2}(G)} \\
& \leq\left\|\chi_{N}\left(\|u\|_{H_{0}^{2}(G)}\right)\left(P_{m}[u, \mathcal{G}[u, u]]-[u, \mathcal{G}[u, u]]\right)\right\|_{L^{2}(G)} \\
& \quad+C_{N}\left\|u-u_{m}\right\|_{H_{0}^{2}(G)}
\end{aligned}
$$


and

$$
\int_{t_{1}}^{t_{2}} 2\left\langle\left[u_{m}, \mathcal{G}\left[u_{m}, u_{m}\right]\right], u_{m t}\right\rangle d t=\left\|\Delta v_{m}\left(t_{2}\right)\right\|_{L^{2}(G)}^{2}-\left\|\Delta v_{m}\left(t_{1}\right)\right\|_{L^{2}(G)}^{2},
$$

where $v_{m}=\mathcal{G}\left[u_{m}, u_{m}\right]$. We now define a stopping time

$$
\tau_{N}=\inf \left\{t>0 \mid\|u(t)\|_{H_{0}^{2}(G)} \geq N\right\} .
$$

By combining these and passing $m \rightarrow \infty$, we arrive at

$$
\begin{aligned}
\left\|u_{t}\left(t_{2}\right)\right\|_{L^{2}(G)}^{2}+\left\|\Delta u\left(t_{2}\right)\right\|_{L^{2}(G)}^{2}+\left\|\Delta v\left(t_{2}\right)\right\|_{L^{2}(G)}^{2} & \\
= & \left\|u_{t}\left(t_{1}\right)\right\|_{L^{2}(G)}^{2}+\left\|\Delta u\left(t_{1}\right)\right\|_{L^{2}(G)}^{2}+\left\|\Delta v\left(t_{1}\right)\right\|_{L^{2}(G)}^{2} \\
& -2 \alpha \int_{t_{1}}^{t_{2}}\left\|u_{t}\right\|_{L^{2}(G)}^{2} d t+2 \sum_{j=1}^{\infty} \int_{t_{1}}^{t_{2}}\left\langle g_{j}, u_{t}\right\rangle d B_{j}+\sum_{j=1}^{\infty} \int_{t_{1}}^{t_{2}}\left\|g_{j}\right\|_{L^{2}(G)}^{2} d t
\end{aligned}
$$

for all $0 \leq t_{1} \leq t_{2} \leq \tau_{N}$ and for almost all $\omega \in \Omega$, where $v=\mathcal{G}[u, u]$. In the same way, we can also derive

$$
\begin{aligned}
\left\langle u_{t}\left(t_{2}\right), u\left(t_{2}\right)\right\rangle+\frac{\alpha}{2}\left\|u\left(t_{2}\right)\right\|_{L^{2}(G)}^{2} & \\
= & \left\langle u_{t}\left(t_{1}\right), u\left(t_{1}\right)\right\rangle+\frac{\alpha}{2}\left\|u\left(t_{1}\right)\right\|_{L^{2}(G)}^{2} \\
& -\int_{t_{1}}^{t_{2}}\left(\|\Delta u\|_{L^{2}(G)}^{2}+\|\Delta v\|_{L^{2}(G)}^{2}-\left\|u_{t}\right\|_{L^{2}(G)}^{2}\right) d t+\sum_{j=1}^{\infty} \int_{t_{1}}^{t_{2}}\left\langle g_{j}, u\right\rangle d B_{j}
\end{aligned}
$$

for all $0 \leq t_{1} \leq t_{2} \leq \tau_{N}$ and for almost all $\omega \in \Omega$. We now write $u_{N}=u$ to signify the dependence of $u$ on $\chi_{N}(\cdot)$. It follows from the Burkholder-Davis-Gundy inequality that

$$
\begin{aligned}
& E\left(\sup _{0 \leq t \leq \tau_{N} \wedge T}\left|\sum_{j=1}^{\infty} \int_{0}^{t}\left\langle g_{j}, u_{N t}\right\rangle d B_{j}\right|\right) \\
& \leq M E\left(\sum_{j=1}^{\infty} \int_{0}^{\tau_{N} \wedge T}\left\|g_{j}\right\|_{L^{2}(G)}^{2}\left\|u_{N t}\right\|_{L^{2}(G)}^{2} d t\right)^{1 / 2} \\
& \quad \leq \delta E\left(\sup _{0 \leq t \leq \tau_{N} \wedge T}\left\|u_{N t}\right\|_{L^{2}(G)}^{2}\right)+\frac{M^{2} T}{4 \delta} \sum_{j=1}^{\infty}\left\|g_{j}\right\|_{L^{2}(G)}^{2}
\end{aligned}
$$

for all $\delta>0$ and for some positive constant $M$ independent of $N$ and $T$. Thus, we can derive from (4.14)

$$
\begin{aligned}
& E\left(\sup _{0 \leq t \leq \tau_{N} \wedge T}\left(\left\|u_{N t}(t)\right\|_{L^{2}(G)}^{2}+\left\|\Delta u_{N}(t)\right\|_{L^{2}(G)}^{2}+\left\|\Delta v_{N}(t)\right\|_{L^{2}(G)}^{2}\right)\right) \\
& \quad \leq C\left(\left\|u_{0}\right\|_{H_{0}^{2}(G)}^{2}+\left\|u_{1}\right\|_{L^{2}(G)}^{2}+\left\|\mathcal{G}\left[u_{0}, u_{0}\right]\right\|_{H_{0}^{2}(G)}^{2}\right)+C T \sum_{j=1}^{\infty}\left\|g_{j}\right\|_{L^{2}(G)}^{2}
\end{aligned}
$$

for some constant $C$ independent of $N$ and $T>0$. Thus, we find that

$$
P\left(\tau_{N} \leq T\right) \leq C_{T} / N^{2} \rightarrow 0 \quad \text { as } N \rightarrow \infty
$$


Since $\tau_{N_{1}} \leq \tau_{N_{2}}$ for $N_{1}<N_{2}$, it follows that

$$
\lim _{N \rightarrow \infty} \tau_{N}=\tau_{\infty} \geq T \quad \text { for almost all } \omega \in \Omega .
$$

Since $T$ is arbitrary,

$$
\tau_{N} \uparrow \infty \quad \text { as } N \rightarrow \infty
$$

for almost all $\omega \in \Omega$. By the pathwise uniqueness of $u_{N}$, we have $u_{N_{1}}=u_{N_{2}}$ on $\left[0, \tau_{N_{1}} \wedge \tau_{N_{2}}\right]$ for almost all $\omega \in \Omega$, and we can define

$$
u(t)=u_{N}(t) \quad \text { for } t \in\left[0, \tau_{N}\right] .
$$

Then, this $u$ is the desired solution. Now (4.8) implies (3.12). Since each $\left(u_{N}, u_{N t}\right)$ is adapted to $\left\{\mathcal{F}_{t}\right\},\left(u, u_{t}\right)$ is adapted to $\left\{\mathcal{F}_{t}\right\}$. By Fatou's lemma, we derive from (4.17) and (4.20) that

$$
\begin{aligned}
& E\left(\sup _{0 \leq t \leq T}\left(\left\|u_{t}(t)\right\|_{L^{2}(G)}^{2}+\|\Delta u(t)\|_{L^{2}(G)}^{2}+\|\Delta v(t)\|_{L^{2}(G)}^{2}\right)\right) \\
& \quad \leq C\left(\left\|u_{0}\right\|_{H_{0}^{2}(G)}^{2}+\left\|u_{1}\right\|_{L^{2}(G)}^{2}+\left\|\mathcal{G}\left[u_{0}, u_{0}\right]\right\|_{H_{0}^{2}(G)}^{2}\right)+C T \sum_{j=1}^{\infty}\left\|g_{j}\right\|_{L^{2}(G)}^{2}
\end{aligned}
$$

for all $T>0$, where $v=\mathcal{G}[u, u]$.

For the proof of pathwise uniqueness, we suppose that $\left(\tilde{u}, \tilde{u}_{t}\right)$ is another solution of $(1.1)-(1.4)$ in $L^{2}\left(\Omega ; C\left([0, T] ; H_{0}^{2}(G) \times L^{2}(G)\right)\right)$. Then, $u-\tilde{u}$ satisfies

$$
u_{t t}-\tilde{u}_{t t}+\alpha\left(u_{t}-\tilde{u}_{t}\right)+\Delta^{2}(u-\tilde{u})+[u, \mathcal{G}[u, u]]-[\tilde{u}, \mathcal{G}[\tilde{u}, \tilde{u}]]=0
$$

for almost all $\omega \in \Omega$. Since $\left(u, u_{t}\right)$ and $\left(\tilde{u}, \tilde{u}_{t}\right)$ belong to $C\left([0, T] ; H_{0}^{2}(G) \times L^{2}(G)\right)$ for almost all $\omega$, we can apply the same argument as for the deterministic case to conclude that $u \equiv \tilde{u}$ for almost all $\omega \in \Omega$. This completes the proof of Theorem 3.1.

Next we will obtain uniform estimates. Fix any $\lambda$ such that

$$
0<\lambda<\min \left(1, \alpha, \lambda_{1}\right),
$$

where $\lambda_{1}$ is the first eigenvalue of (3.1), and define

$$
\begin{aligned}
Q(t)= & \left\|u_{t}(t)\right\|_{L^{2}(G)}^{2}+\|\Delta u(t)\|_{L^{2}(G)}^{2}+\|\Delta v(t)\|_{L^{2}(G)}^{2} \\
& +\lambda\left\langle u_{t}(t), u(t)\right\rangle+\frac{\alpha}{2} \lambda\|u(t)\|_{L^{2}(G)}^{2} .
\end{aligned}
$$

By virtue of (4.20) and (4.21), $u$ satisfies (4.14) and (4.15) for all $0 \leq t_{1}<t_{2}<\infty$ and for almost all $\omega$. Since integrability is guaranteed by (4.22), it follows from (4.14) and (4.15) that

$$
\begin{aligned}
E\left(Q\left(t_{2}\right)\right)-E\left(Q\left(t_{1}\right)\right)= & -\lambda \int_{t_{1}}^{t_{2}} E\left(\|\Delta u\|_{L^{2}(G)}^{2}+\|\Delta v\|_{L^{2}(G)}^{2}\right) d t \\
& -(2 \alpha-\lambda) \int_{t_{1}}^{t_{2}} E\left(\left\|u_{t}\right\|_{L^{2}(G)}^{2}\right) d t+\int_{t_{1}}^{t_{2}} \sum_{j=1}^{\infty}\left\|g_{j}\right\|_{L^{2}(G)}^{2} d t
\end{aligned}
$$


for all $0 \leq t_{1}<t_{2}<\infty$. We can derive

$$
\frac{d}{d t} E(Q(t)) \leq-c E(Q(t))+\sum_{j=1}^{\infty}\left\|g_{j}\right\|_{L^{2}(G)}^{2}
$$

for all $t>0$, where $c$ is a positive constant depending on $\alpha, \lambda_{1}$, and $\lambda$. This yields

$$
E(Q(t)) \leq C_{M} \text { for all } t \geq 0,
$$

where $M$ is a constant such that $Q(0) \leq M$, and $C_{M}$ is a constant depending on $M$ and the last term of (4.27). By virtue of (4.24), this yields (2.2).

According to the above argument for the existence of solutions, we could take any $s \geq 0$ as the initial time and $\zeta=\left(\zeta_{0}, \zeta_{1}\right)$ as the initial value for the Cauchy problem $(1.1)-(1.3)$ if $\zeta$ is $H_{0}^{2}(G) \times L^{2}(G)$-valued $\mathcal{F}_{s}$-measurable such that $\zeta \in L^{2}\left(\Omega ; H_{0}^{2}(G) \times\right.$ $\left.L^{2}(G)\right)$, and $\mathcal{G}\left[\zeta_{0}, \zeta_{0}\right] \in L^{2}\left(\Omega ; H_{0}^{2}(G)\right)$. We now write $X(t, s ; \zeta)=\left(u, u_{t}\right)$, where $u$ is the solution of (1.1)-(1.3) for $t \geq s$ satisfying $\left(u(s), u_{t}(s)\right)=\zeta$. Then, $X(\cdot, s ; \zeta) \in$ $L^{2}\left(\Omega ; C\left([s, T] ; H_{0}^{2}(G) \times L^{2}(G)\right)\right)$ for all $T>s$, and (4.28) holds for all $t \geq s$. For each $0 \leq s<t, z \in H_{0}^{2}(G) \times L^{2}(G)$, and $\Gamma \in \mathcal{B}\left(H_{0}^{2}(G) \times L^{2}(G)\right)$, we set as in (2.1)

$$
\mathcal{P}(s, z ; t, \Gamma)=P(X(t, s ; z) \in \Gamma) .
$$

Lemma 4.1. Choose any bounded continuous function $\psi$ on $H_{0}^{2}(G) \times L^{2}(G)$, $t>s \geq 0$. Then,

$$
E(\psi(X(t, s ; z)))=\int_{H_{0}^{2}(G) \times L^{2}(G)} \mathcal{P}(s, z ; t, d y) \psi(y)
$$

is continuous in $z \in H_{0}^{2}(G) \times L^{2}(G)$.

Proof. Let $\left\{z_{n}\right\}_{n=1}^{\infty}$ be a sequence in $H_{0}^{2}(G) \times L^{2}(G)$ such that $z_{n} \rightarrow z$ in $H_{0}^{2}(G) \times$ $L^{2}(G)$. Let us fix any $t>s \geq 0$. By (4.22), we have

$$
\left\{\begin{array}{l}
E\left(\sup _{s \leq \eta \leq t}\|X(\eta, s ; z)\|_{H_{0}^{2}(G) \times L^{2}(G)}\right) \leq M, \\
E\left(\sup _{s \leq \eta \leq t}\left\|X\left(\eta, s ; z_{n}\right)\right\|_{H_{0}^{2}(G) \times L^{2}(G)}\right) \leq M \quad \text { for all } n \geq 1
\end{array}\right.
$$

for some positive constant $M$. Let us fix any $\epsilon>0$ and any bounded continuous function $\psi$ on $H_{0}^{2}(G) \times L^{2}(G)$. Since $H_{0}^{2}(G) \times L^{2}(G)$ is a Polish space and $P(X(t, s ; z) \in \cdot)$ is a probability measure over $\left\{H_{0}^{2}(G) \times L^{2}(G), \mathcal{B}\left(H_{0}^{2}(G) \times L^{2}(G)\right)\right\}$, there is a compact subset $\mathcal{K}$ of $H_{0}^{2}(G) \times L^{2}(G)$ such that

$$
P(X(t, s ; z) \in \mathcal{K})>1-\epsilon .
$$

By virtue of (4.30), there is some $R>0$ such that

$$
\left\{\begin{array}{l}
P\left(\sup _{s \leq \eta \leq t}\left\|X\left(\eta, s ; z_{n}\right)\right\|_{H_{0}^{2}(G) \times L^{2}(G)} \leq R\right)>1-\epsilon \quad \text { for all } n \geq 1 \\
P\left(\sup _{s \leq \eta \leq t}\|X(\eta, s ; z)\|_{H_{0}^{2}(G) \times L^{2}(G)} \leq R\right)>1-\epsilon
\end{array}\right.
$$

By taking $R$ larger, we also have

$$
\mathcal{K} \subset\left\{y \in H_{0}^{2}(G) \times L^{2}(G) \mid\|y\|_{H_{0}^{2}(G) \times L^{2}(G)} \leq R\right\} .
$$


Let us fix such $R$ and write for each $n$

$$
\begin{aligned}
A_{n}= & \left\{\sup _{s \leq \eta \leq t}\left\|X\left(\eta, s ; z_{n}\right)\right\|_{H_{0}^{2}(G) \times L^{2}(G)} \leq R\right\} \\
& \bigcap\left\{\sup _{s \leq \eta \leq t}\|X(\eta, s ; z)\|_{H_{0}^{2}(G) \times L^{2}(G)} \leq R\right\} \\
& \bigcap\{X(t, s ; z) \in \mathcal{K}\} .
\end{aligned}
$$

We will estimate the integral on the right-hand side of

$$
\begin{aligned}
& \left|E(\psi(X(t, s ; z)))-E\left(\psi\left(X\left(t, s ; z_{n}\right)\right)\right)\right| \\
& \quad \leq \int_{A_{n}}\left|\psi(X(t, s ; z))-\psi\left(X\left(t, s ; z_{n}\right)\right)\right| d P+6 M \epsilon,
\end{aligned}
$$

where $M$ is a positive constant such that $|\psi(y)| \leq M$ for all $y$. By means of (3.9), we can derive from (4.23) that

$$
\left\|X(t, s ; z)-X\left(t, s ; z_{n}\right)\right\|_{H_{0}^{2}(G) \times L^{2}(G)}^{2} \leq C_{R}\left\|z_{n}-z\right\|_{H_{0}^{2}(G) \times L^{2}(G)}^{2}
$$

for all $\omega \in \tilde{A}_{n}$, where $\tilde{A}_{n} \subset A_{n}$ and $P\left(A_{n} \backslash \tilde{A}_{n}\right)=0$, and $C_{R}$ is a constant independent of $n$. Since $\psi$ is continuous on $H_{0}^{2}(G) \times L^{2}(G)$ and $\mathcal{K}$ is compact, there is $\delta>0$ such that

$$
|\psi(x)-\psi(y)|<\epsilon
$$

for every $x \in \mathcal{K}, y \in H_{0}^{2}(G) \times L^{2}(G)$ satisfying $\|x-y\|_{H_{0}^{2}(G) \times L^{2}(G)}<\delta$. Hence, it follows from (4.36) that there is $N \geq 1$ such that for all $n \geq N$,

$$
\int_{A_{n}}\left|\psi(X(t, s ; z))-\psi\left(X\left(t, s ; z_{n}\right)\right)\right| d P<\epsilon,
$$

which yields

$$
\left|E(\psi(X(t, s ; z)))-E\left(\psi\left(X\left(t, s ; z_{n}\right)\right)\right)\right|<\epsilon+6 M \epsilon
$$

for all $n \geq N$. Thus, $E(\psi(X(t, s ; z)))$ is continuous in $z$.

This implies that $\mathcal{P}(s, \cdot ; t, \Gamma)$ is $\mathcal{B}(\Xi)$-measurable for all $0 \leq s<t<\infty$ and $\Gamma \in \mathcal{B}(\Xi)$. This can be seen by the same argument as in the previous proof of the measurability of $P(X(\cdot, 0 ; z))$.

Lemma 4.2. $X(\cdot)$ has the Markov property, and its transition probability function is time-homogeneous.

Proof. By the uniqueness of solution, it holds that for any $0 \leq r<s<t$ and $z \in H_{0}^{2}(G) \times L^{2}(G)$,

$$
X(t, r ; z)=X(t, s ; X(s, r ; z))
$$

for almost all $\omega$. We have to show that

$$
E\left(\psi(X(t, s ; X(s, r ; z))) \mid \mathcal{F}_{s}\right)=\mathcal{P}_{s, t}(\psi)(X(s, r ; z))
$$

for almost all $\omega$, for each bounded continuous function $\psi$ on $H_{0}^{2}(G) \times L^{2}(G)$, where

$$
\mathcal{P}_{s, t} \psi(y)=E(\psi(X(t, s ; y))) .
$$


According to the proof of Theorem 3.1, the solution was obtained by the truncation method. Let $X_{N}=X_{N}(t, s ; \zeta)$ denote the solution $\left(u_{N}, \partial_{t} u_{N}\right)$ of (4.3)-(4.4) satisfying $\left(u_{N}(s), \partial_{t} u_{N}(s)\right)=\zeta$, where $\zeta=\left(\zeta_{0}, \zeta_{1}\right)$ is $H_{0}^{2}(G) \times L^{2}(G)$-valued $\mathcal{F}_{s}$-measurable such that $\zeta \in L^{2}\left(\Omega ; H_{0}^{2}(G) \times L^{2}(G)\right)$ and $\mathcal{G}\left[\zeta_{0}, \zeta_{0}\right] \in L^{2}\left(\Omega ; H_{0}^{2}(G)\right)$. Then, we know that for each $T>s$,

$$
X(t, s ; \zeta)=\lim _{N \rightarrow \infty} X_{N}(t, s ; \zeta) \quad \text { in } C\left([s, T] ; H_{0}^{2}(G) \times L^{2}(G)\right)
$$

for almost all $\omega$. For each $N \geq 1$ and each bounded continuous function $\psi$ on $H_{0}^{2}(G) \times$ $L^{2}(G)$, it holds that

$$
E\left(\psi\left(X_{N}(t, s ; \zeta)\right) \mid \mathcal{F}_{s}\right)=\mathcal{P}_{s, t}^{N}(\psi)(\zeta)
$$

for almost all $\omega$, which follows directly from the argument in [6, p. 250]. Here $\mathcal{P}_{s, t}^{N}$ is defined by

$$
\mathcal{P}_{s, t}^{N} \psi(y)=E\left(\psi\left(X_{N}(t, s ; y)\right)\right)
$$

Since $\psi$ is a bounded continuous function, we pass $N \rightarrow \infty$ to arrive at

$$
E\left(\psi(X(t, s ; \zeta)) \mid \mathcal{F}_{s}\right)=P_{s, t}(\psi)(\zeta)
$$

for almost all $\omega$. Hence $X(\cdot)$ has the Markov property.

Since $g_{j}$ 's are independent of time, we can apply the result in $[6$, p. 251] to see that the transition probability function is time-homogeneous.

Lemma 4.3. Let $S_{L}=\left\{y \in H_{0}^{2}(G) \times L^{2}(G) \mid\|y\|_{H_{0}^{2}(G) \times L^{2}(G)} \leq L\right\}$, and let $\left\{z_{n}\right\}_{n=1}^{\infty}$ be a sequence in $S_{L}$ such that $z_{n} \rightarrow z$ in $H_{0}^{1}(G) \times H^{-1}(G)$. If $\phi$ is a bounded continuous function on $H_{0}^{1}(G) \times H^{-1}(G)$, then for each $t>0$,

$$
E\left(\phi\left(X\left(t, 0 ; z_{n}\right)\right)\right) \rightarrow E(\phi(X(t, 0 ; z)))
$$

as $z_{n} \rightarrow z$ in $H_{0}^{1}(G) \times H^{-1}(G)$.

Proof. Let us fix any $t^{*}>0$, and write

$$
Y_{n}(t)=X\left(t, 0 ; z_{n}\right)-X(t, 0 ; z) .
$$

Suppose that

$$
\left\|X\left(t, 0 ; z_{n}\right)\right\|_{H_{0}^{2}(G) \times L^{2}(G)} \leq R, \quad\|X(t, 0 ; z)\|_{H_{0}^{2}(G) \times L^{2}(G)} \leq R
$$

for all $0 \leq t \leq t^{*}$ for some constant $R$. It follows from (4.23) and the basic inequality established in [1] that

$$
\begin{aligned}
& \left\|Y_{n}\left(t_{2}\right)\right\|_{H_{0}^{1}(G) \times H^{-1}(G)}^{2}-\left\|Y_{n}\left(t_{1}\right)\right\|_{H_{0}^{1}(G) \times H^{-1}(G)}^{2} \\
& \quad \leq C_{1} \log \left(1+\lambda_{N}\right) \int_{t_{1}}^{t_{2}}\left\|Y_{n}(s)\right\|_{H_{0}^{1}(G) \times H^{-1}(G)}^{2} d s+C_{2} t^{*} \lambda_{N+1}^{-\beta}
\end{aligned}
$$

for all $0 \leq t_{1}<t_{2} \leq t^{*}$, all $N \geq N_{0}$, for some constant $0<\beta<1$, and for positive integer $N_{0}$. Here $\lambda_{N}$ is the $N$ th eigenvalue of (3.1), and $C_{1}$ and $C_{2}$ are positive constants depending only on $\beta$ and $R$. We partition $\left[0, t^{*}\right]$ such that

$$
0=t_{0}<t_{1}<\cdots<t_{K}=t^{*}, \quad t_{k}-t_{k-1}=t^{*} / K<\beta / C_{1}, \quad 1 \leq k \leq K .
$$


By the Gronwall inequality, we can derive from (4.48) that

$$
\begin{aligned}
& \left\|Y_{n}(t)\right\|_{H_{0}^{1}(G) \times H^{-1}(G)}^{2} \\
& \quad \leq\left(\left\|Y_{n}\left(t_{k}\right)\right\|_{H_{0}^{1}(G) \times H^{-1}(G)}^{2}+C_{2} t^{*} \lambda_{N+1}^{-\beta}\right)\left(1+\lambda_{N}\right)^{C_{1}\left(t-t_{k}\right)}
\end{aligned}
$$

for all $t \in\left[t_{k}, t_{k+1}\right]$, all $N \geq N_{0}$, and for each $k=0, \ldots, K-1$. Since $\lambda_{N} \uparrow \infty$ as $N \rightarrow \infty$, we use (4.49) to infer from (4.50) that for given $\epsilon>0$, there is $\epsilon_{K}>0$ such that if $\left\|Y_{n}\left(t_{K-1}\right)\right\|_{H_{0}^{1}(G) \times H^{-1}(G)}<\epsilon_{K}$,

$$
\left\|Y_{n}\left(t_{K}\right)\right\|_{H_{0}^{1}(G) \times H^{-1}(G)}<\epsilon .
$$

By iteration, we find that there is $\epsilon_{1}>0$ such that if $\left\|z_{n}-z\right\|_{H_{0}^{1}(G) \times H^{-1}(G)}<\epsilon_{1}$, (4.51) holds. By the same argument as in the proof of Lemma 4.1, we arrive at (4.45).

LEMma 4.4. Let $\psi$ be a bounded continuous function on $H_{0}^{2}(G) \times L^{2}(G)$. Then, there is a sequence $\left\{\psi_{k}\right\}_{k=1}^{\infty}$ such that each $\psi_{k}$ is a continuous function on $H_{0}^{1}(G) \times$ $H^{-1}(G)$ bounded uniformly in $k$, and

$$
\psi_{k}(y) \rightarrow \psi(y) \quad \text { as } k \rightarrow \infty
$$

for each $y \in H_{0}^{2}(G) \times L^{2}(G)$.

Proof. It is enough to set

$$
\psi_{k}(y)=\psi\left(\left(P_{k} y_{1}, P_{k} y_{2}\right)\right) \quad \text { for } y=\left(y_{1}, y_{2}\right) \in H_{0}^{1}(G) \times H^{-1}(G), k=1,2, \ldots,
$$

where $P_{k}$ is the projection onto the subspace spanned by $\left\{\phi_{1}, \ldots, \phi_{k}\right\}$.

Finally, we set

$$
\Xi=H_{0}^{2}(G) \times L^{2}(G), \quad \Upsilon=H_{0}^{1}(G) \times H^{-1}(G) .
$$

Then, assumptions (I)-(V) follow from the above lemmas, and the proof of Theorem 3.2 is complete.

\section{REFERENCES}

[1] A. Boutet De Monvel And I. Chueshov, Uniqueness theorem for weak solutions of von Karman evolution equations, J. Math. Anal. Appl., 221 (1998), pp. 419-429.

[2] P.L. ChOw And R.Z. KhasminskiI, Stationary solutions of nonlinear stochastic evolution equations, Stochastic Anal. Appl., 15 (1997), pp. 671-699.

[3] I. CHUESHOv, Existence of statistical solutions of a stochastic system of von Karman equations in a bounded domain, Sb. Math., 50 (1985), pp. 279-298.

[4] I. Chueshov, Strong solutions and the attractor of the von Karman equations, Sb. Math., 69 (1991), pp. 25-36.

[5] I. Chueshov and I. Lasiecka, Inertial manifolds for von Karman plate equations, Appl. Math. Optim., 46 (2002), pp. 179-206.

[6] G. Da Prato and J. Zabczyk, Stochastic Equations in Infinite Dimensions, Cambridge University Press, Cambridge, 1992.

[7] G. Da Prato and J. Zabczyk, Ergodicity for Infinite Dimensional Systems, Cambridge University Press, Cambridge, 1996.

[8] A. Favini, M. Horn, I. Lasiecka, and D. Tataru, Global existence, uniqueness and regularity of solutions to a von Karman system with nonlinear boundary dissipation, Differential Integral Equations, 9 (1996), pp. 267-294.

[9] A. Favini, M. Horn, I. Lasiecka, and D. Tataru, Addendum to the paper: Global existence, uniqueness and regularity of solutions to a von Karman system with nonlinear boundary dissipation, Differential Integral Equations, 10 (1997), pp. 197-200. 
[10] V.I. Gishlarkaev, The existence of statistical solutions of the stochastic von Karman system in a bounded domain, Math. Notes, 58 (1995), pp. 692-702.

[11] I. Karatzas and S. Shreve, Brownian Motion and Stochastic Calculus, 2nd ed., Springer, New York, Berlin, Heidelberg, 1997.

[12] N. Krylov and N. Bogolyubov, La théorie générale de la mesure dans son application à l'étude des systémes de la Mécanique non linéaire, Ann. of Math. (2), 38 (1937), pp. 65-113.

[13] P. Kotelenez, A submartingale type inequality with applications to stochastic evolution equations, Stochastics, 8 (1982), pp. 139-151.

[14] G. LEHA AND G. RITTER, Lyapunov-type conditions for stationary distributions of diffusion processes on Hilbert spaces, Stochastics Stochastics Rep., 48 (1994), pp. 195-225.

[15] J.L. Lions, Quelques Méthodes de Résolution des Problémes aux Limites Non Linéaires, Dunod, Paris, 1969.

[16] B. Maslowski And J. Seidler, On sequentially weakly Feller solutions to SPDE's, Atti Accad. Naz. Lincei Cl. Sci. Fis. Mat. Natur. Rend. Lincei (9) Mat. Appl., 10 (1999), pp. 69-78.

[17] B. MASLOWSKI AND J. SeIDler, Strong Feller solutions to SPDE's are strong Feller in the weak topology, Studia Math., 148 (2001), pp. 111-129. 